\title{
Physicochemical properties and crystallisation behaviour of bakery shortening produced from stearin fraction of palm-based diacyglycerol blended with various vegetable oils
}

\begin{abstract}
The stearin fraction of palm-based diacylglycerol (PDAGS) was produced from dry fractionation of palm-based diacylglycerol (PDAG). Bakery shortening blends were produced by mixing PDAGS with either palm mid fraction, PMF (PDAGS/PMF), palm olein, POL(PDAGS/POL) or sunflower oil, SFO (PDAGS/SFO) at PDAGS molar fraction of XPDAGS $=0.4 \%, 0.5 \%, 0.6 \%, 0.7 \%, 0.8 \%, 0.9 \%$. The physicochemical results obtained indicated that $\mathrm{C} 16: 0$ and $\mathrm{C} 18: 1$ were the dominant fatty acids for PDAGS/PMF and PDAGS/POL, while C18:1 and C18:2 were dominant in the PDAGS/SFO mixtures. SMP and SFC of the PDAGS were reduced with the addition of PMF, POL and SFO. Binary mixtures of PDAGS/PMF had better structural compatibility and full miscibility with each other. PDAGS/PMF and PDAGS/SFO crystallised in 6AjJ polymorphs in the presence of $0.410 .5 \%$ PDAGS while PDAGS/POL resulted in 6 polymorphs crystal. The results gave indication that PDAGS: PMF at 50\%:50\% and 60\%:40\% (w/w) were the most suitable fat blend to be used as bakery shortening.
\end{abstract}

Keyword: Diacylglycerol fractions; Shortening; Stearin; Palm 